Psychother Psychosom 2020;89:109-110

DOI: $10.1159 / 000502918$

\section{Effectiveness of Acute-Phase Treatment of Depression Is Not Influenced by Comorbid Personality Disorders: Results from a Meta-Analysis and Meta-Regression}

\author{
Suzanne C. van Bronswijk ${ }^{\mathrm{a}, \mathrm{b}}$ Eva Maria Köster ${ }^{\mathrm{c}}$ \\ Frenk P.M.L. Peeters ${ }^{\mathrm{C}}$ \\ ${ }^{a}$ Department of Psychiatry and Psychology, Maastricht \\ University Medical Center+, Maastricht, The Netherlands; \\ ${ }^{b}$ School for Mental Health and Neuroscience, Faculty of Health, \\ Medicine and Life Sciences, Maastricht University, Maastricht, \\ The Netherlands; ${ }^{C}$ Faculty of Psychology and Neuroscience, \\ Maastricht University, Maastricht, The Netherlands
}

\section{Dear Editor,}

About $45 \%$ of the individuals suffering from major depressive disorder (MDD) also meet criteria for a personality disorder (PD), falling predominantly in the DSM-based cluster C. This comorbidity is regarded clinically relevant as it is accompanied by lower levels of daily functioning, and is often considered to be a predictor of poor acute-phase treatment response in $\operatorname{MDD}[1,2]$. However, the evidence that the presence of a comorbid PD may have a negative impact on acute-phase treatment outcomes for MDD is not robust and biased by unstructured PD and MDD assessments, naturalistic treatment allocation, not controlling for significant confounders (e.g., earlier onset, childhood adversity, and episode duration), and no consideration of baseline depression severity differences between individuals with and without comorbid PD [3]. As the last meta-analysis [2] included only studies published before 2007, and given the methodological considerations above [3], we conducted a meta-analysis and meta-regression with strict inclusion criteria to maximize validity of the results. For that purpose, we collected only well-designed controlled trials that investigated the impact of comorbid PD on acute-phase treatment outcome for MDD with the use of (semi-) structured interviews for both MDD and PD. Additionally, the primary outcome was depression severity change during treatment, thereby avoiding bias following from baseline severity differences between individuals with and without comorbid PD.

A database search up to April 3, 2017, was conducted in PubMed, PsycINFO, and Embase (study protocol: PROSPERO, CRD42019120200). References of selected articles, earlier reviews, and meta-analyses were searched. Treatment studies for MDD with a subset of individuals with comorbid PD were included. MDD and PD diagnoses had to be based on pre-treatment structured interviews. Primary outcome was depression severity change during treatment, and secondary outcomes were response and remission rates. Data was extracted from published records, and corresponding authors were contacted to provide additional information. Effect sizes (Hedges' $g$ effect sizes, risk ratios [RRs] and odds ratios [ORs]) were estimated with random-effects models. Included studies were evaluated with the Risk of Bias tool. Study-level variables were examined using meta-regression. Publication bias was assessed using funnel plots and Egger's test.

A total of 11 studies were identified, of which 6 studies $(12 \mathrm{com}$ parisons) with 942 individuals (447 with a comorbid PD) were included in the meta-analysis [4-9]. Trials were conducted in outpatient settings in the Netherlands $(n=1)$, Iran $(n=1)$, the United States $(n=3)$, and the United Kingdom $(n=1)$. Treatment consisted of different types of psychotherapy (cognitive [behavioral] therapy, interpersonal psychotherapy, behavioral activation, psychodynamic interpersonal psychotherapy, 8 conditions, $n=350$,) and antidepressant medication (sertraline, paroxetine, nortriptyline, fluoxetine, 4 conditions, $n=592$ ). Change in depression severity was measured with self-report questionnaires (Beck Depression Inventory, BDI), clinician-rated measures (Hamilton Depression Rating Scale; HDRS), or with both. Response rates were available for 4 studies $[4,6-8]$. These were defined as (i) at least $50 \%$ reduction, or (ii) a predefined minimum decrease on the severity scale, or a combination of both criteria. Remission rates were available for 4 studies [4, $6,8,9]$ and were based on cutoff scores on depression severity scales (HDRS $\leq 7$, BDI $\leq 9,10$ or 15 ). As shown in Figure 1 , the mean pooled Hedges' $g$ effect size was $g=0.03$ ( $95 \%$ CI -0.15 to $0.20, p=$ 0.27 ), indicating no significant difference in the average depression severity change between individuals with and without a PD. The heterogeneity of the pooled effect size was low with a moderately high level of uncertainty $\left(\mathrm{I}^{2}=17.6 \%, 95 \%\right.$ CI $\left.0.00 \%-56.95 \%\right)$. A total of 219 individuals with a PD achieved response (58.2\%), compared to 209 individuals without a PD (54.5\%); the pooled RR for response was $1.14(95 \% \mathrm{CI} 0.99-1.31, p=0.07)$, and the pooled OR was 1.32 (95\% CI $0.83-2.12, p=0.24)$, indicating no significant difference between individuals with and without a PD. A total of 98 individuals with a PD met criteria for remission (44.3\%), and 125 individuals without PD achieved remission (47.2\%); the pooled RR for remission was 0.90 (95\% CI $0.74-1.09, p=0.27)$, and the pooled OR was 0.75 (95\% CI $0.47-1.19, p=0.23)$, indicating no significant difference between individuals with and without a PD. Risk of bias was low. The meta-regression did not indicate significant associations between specific study-level variables and between-study heterogeneity of the results; however, these analyses are highly exploratory due to missing data and a low number of included studies. There was no indication for publication bias.

Findings indicate no significant difference between individuals with and without a comorbid PD in terms of average depression severity change, response rates, and remission rates. Our findings do not concur with results from the most recent previous metaanalyses $[1,2]$. However, in contrast to these meta-analyses, we included only controlled studies in which diagnoses were based on (semi-) structured interviews for both mood disorder and personality to reduce heterogeneity and biases, and maximize the validity of the results. These stringent inclusion criteria did, however, result in a low number of included studies, which can be considered as a limitation. Other limitations are the lack of dimensional assessment of personality and long-term treatment outcomes.

\section{KARGER}

karger@karger.com www.karger.com/pps

\section{() 2019 The Author(s) \\ Published by S. Karger AG, Basel \\ Karger}

Open access

This article is licensed under the Creative Commons AttributionNonCommercial-NoDerivatives 4.0 International License (CC BY NC-ND) (http://www.karger.com/Services/OpenAccessLicense). Usage and distribution for commercial purposes as well as any distribution of modified material requires written permission.
Suzanne C. van Bronswijk

Department of Psychiatry and Neuropsychology

Maastricht University Medical Center+, P. Debyelaan 25/Postbus 5800

NL-6202 AZ Maastricht (The Netherlands)

E-Mail suzanne.vanbronswijk@maastrichtuniversity.nl 


\begin{tabular}{|c|c|c|c|c|c|c|c|c|c|c|}
\hline Study & $\mathrm{PD}, n$ & No PD & & & & & & & & Hedges' $g(95 \% \mathrm{Cl})$ \\
\hline Lemmens et al. [4], CT & 26 & 41 & & & & & $\longrightarrow$ & - & & $0.56(0.06-1.06)$ \\
\hline Lemmens et al. [4], IPT & 19 & 45 & & & & 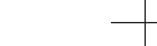 & $\longrightarrow$ & & & $0.39(-0.15-0.93)$ \\
\hline Moradveisi et al. [5], BA & 6 & 39 & & & & - & & & & $-0.47(-2.01-1.06)$ \\
\hline Moradveisi et al. [5], SERT & 5 & 30 & & & & & & & & $-0.02(-1.65-1.61)$ \\
\hline Fournier et al. [6], CT & 27 & 33 & & & & 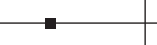 & - & & & $-0.45(-1.0-0.11)$ \\
\hline Fournier et al. [6], PAR & 59 & 61 & & & & 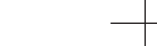 & $\longrightarrow$ & & & $0.19(-0.17-0.56)$ \\
\hline Papakostas et al. [7] & 35 & 24 & & & & $\longrightarrow$ & & & & $-0.16(-0.68-0.36)$ \\
\hline Fava et al. [8] & 243 & 135 & & & & $\square$ & - & & & $-0.06(-0.27-0.15)$ \\
\hline Hardy et al. [9], CBT 8 sessions & 7 & 22 & & & & - & & & & $-0.47(-1.33-0.39)$ \\
\hline Hardy et al. [9], CBT 16 sessions & 7 & 21 & & & & 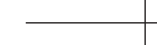 & & & & $0.29(-0.57-1.15)$ \\
\hline Hardy et al. [9], PI 8 sessions & 6 & 23 & & & & & & & & $0.04(-0.86-0.94)$ \\
\hline Hardy et al. [9], PI 8 sessions & 7 & 21 & & & & & & & & $0.27(-1.13-0.59)$ \\
\hline \multirow[t]{3}{*}{ Overall } & & & & & & - & & & & $0.03(-0.15-0.20)$ \\
\hline & & & $\begin{array}{r}r .0 \\
-2.0\end{array}$ & -1.5 & -1.0 & -0.5 & 0.5 & 1.0 & 1.5 & \\
\hline & \multicolumn{6}{|c|}{ Individuals without PD did better } & Individual & th PD & $\overrightarrow{\text { better }}$ & \\
\hline
\end{tabular}

Fig. 1. Effects of comorbid PD on Hedges' $g$ effect sizes. PD, personality disorder; CI, confidence interval; CT, cognitive therapy; IPT, interpersonal psychotherapy; BA, behavioral activation; SERT, sertraline; PAR, paroxetine; CBT, cognitive behavioral therapy; PI, psychodynamic interpersonal psychotherapy.

Based on the findings from the current meta-analysis, we strongly suggest that depressed patients with comorbid PD receive evidence-based treatments following guidelines for their MDD; their acute-phase treatment outcome appears not worse than that of individuals with MDD without comorbid PD. More high-quality studies are needed to establish the effects of comorbid PD for specific depression treatments (differential treatment effects), different types of personality pathology, and long-term outcomes. In addition, disentangling the complex relationships between MDD and PD is and will remain a challenging task for researchers and clinicians alike: How do depression and personality relate to each other, and, to what degree are they really distinct [10]? In future studies, MDD and PD should be reliably, frequently, and concurrently assessed to further understand their interrelationship, and their "state" (current illness) and "trait" (stable set of characteristics) aspects.

\section{Acknowledgement}

We would like to thank all researchers who gave us access to additional information from the included trials.

\section{Disclosure Statement}

The authors have no conflicts of interest to declare.

\section{Funding Sources}

The authors received no specific funding for this work.

\section{Author Contributions}

S.C.B., E.M.K. and F.P.M.L.P. equally contributed to the conception of the design and data collection. Data analysis was performed by S.C.B., and the interpretation of this analysis was done by S.C.B. and F.P.M.L.P. S.C.B. and F.P.M.L.P. wrote the first draft of the paper, E.M.K. provided a critical revision of this draft. All authors gave their final approval of the version to be published.

\section{References}

1 Newton-Howes G, Tyrer P, Johnson T. Personality disorder and the outcome of depression: meta-analysis of published studies. Br J Psychiatry. 2006 Jan;188(1):13-20.

2 Newton-Howes G, Tyrer P, Johnson T, Mulder R, Kool S, Dekker J, et al. Influence of personality on the outcome of treatment in depression: systematic review and meta-analysis. J Pers Disord. 2014 Aug;28(4):577-93.

3 Mulder RT. Personality pathology and treatment outcome in major depression: a review. Am J Psychiatry. 2002 Mar;159(3):359-71.

4 Lemmens LH, Arntz A, Peeters F, Hollon SD, Roefs A, Huibers MJ. Clinical effectiveness of cognitive therapy v. interpersonal psychotherapy for depression: results of a randomized controlled trial. Psychol Med. 2015 Jul;45(10):2095-110.

5 Moradveisi L, Huibers MJ, Renner F, Arasteh M, Arntz A. The influence of comorbid personality disorder on the effects of behavioural activation vs. antidepressant medication for major depressive disorder: results from a randomized trial in Iran. Behav Res Ther. 2013 Aug;51(8):499-506.

6 Fournier JC, DeRubeis RJ, Shelton RC, Gallop R, Amsterdam JD, Hollon $\mathrm{SD}$. Antidepressant medications v. cognitive therapy in people with depression with or without personality disorder. Br J Psychiatry. 2008 Feb; 192(2):124-9.

7 Papakostas GI, Petersen TJ, Farabaugh AH, Murakami JL, Pava JA, Alpert JE, et al. Psychiatric comorbidity as a predictor of clinical response to nortriptyline in treatment-resistant major depressive disorder. J Clin Psychiatry. 2003 Nov;64(11):1357-61.

8 Fava M, Farabaugh AH, Sickinger AH, Wright E, Alpert JE, Sonawalla S, et al. Personality disorders and depression. Psychol Med. 2002 Aug; 32(6): 1049-57.

9 Hardy GE, Barkham M, Shapiro DA, Stiles WB, Rees A, Reynolds S. Impact of Cluster $C$ personality disorders on outcomes of contrasting brief psychotherapies for depression. J Consult Clin Psychol. 1995 Dec;63(6): 997-1004.

10 Klein DN, Kotov R, Bufferd SJ. Personality and depression: explanatory models and review of the evidence. Annu Rev Clin Psychol. 2011;7(1): 269-95. 